\title{
The nature of the ultrafast magnetic phase transition in nickel revealed by correlating EUV- MOKE and ARPES spectroscopies
}

\author{
Zhensheng Tao ${ }^{1,2^{*}}$, Wenjing You ${ }^{1}$, Phoebe Tengdin ${ }^{1}$, Cong Chen ${ }^{1}$, Xun $\mathrm{Shi}^{1}$, Dmitriy \\ Zusin $^{1}$, Yingchao Zhang ${ }^{1}$, Christian Gentry ${ }^{1}$, Adam Blonsky ${ }^{1}$, Mark Keller ${ }^{3}$, Peter \\ Openeer $^{4}$, Henry Kapteyn ${ }^{1}$ and Margaret Murnane ${ }^{1}$ \\ ${ }^{1}$ Department of Physics and JILA, University of Colorado and NIST, Boulder, Colorado 80309, \\ United States \\ ${ }^{2}$ State Key Laboratory of Surface Physics, Department of Physics, Fudan University, Shanghai \\ 200438, People's Republic of China \\ ${ }^{3}$ National Institute of Standards and Technology (NIST), 325 Broadway, Boulder, Colorado 80305, \\ United States \\ ${ }^{4}$ Department of Physics and Astronomy, Uppsala University, Box 516, 75120 Uppsala, Sweden
}

\begin{abstract}
By correlating time- and angle-resolved photoemission (TrARPES) and time-resolved transverse- magneto-optical Kerr effect ( $\mathrm{Tr}-$ TMOKE) measurements, both at extreme ultraviolet (EUV) wavelengths, we uncover the nature of the ultrafast photoinduced magnetic phase transition in Ni. This allows us to explain the ultrafast magnetic response of $\mathrm{Ni}$ at all laser fluences - from a small reduction of the magnetization at low laser fluences, to complete quenching at high laser fluences. We provide an alternative explanation to the fluence-dependent recovery timescales commonly observed in ultrafast magneto-optical spectroscopies on ferromagnets: it is due to the bulk-averaging effect and different depths of sample exhibit distinct dynamics, depending on whether a magnetic phase transition is induced. We also show evidence of two competing channels with two distinct timescales in the recovery process, that suggest the presence of coexisting phases in the material.
\end{abstract}

\section{Introduction}

Magnetization in transition-metal ferromagnets can be strongly suppressed by ultrafast laser irradiation on femtosecond timescales [1]. Despite extensive experimental efforts, the underlying microscopic mechanisms that drive ultrafast magnetization dynamics are still under debate.

In a recent work, using Tr-ARPES, we revealed the existence of critical phenomena during ultrafast demagnetization in Ni [2]. Given this new understanding, it is now essential to revisit results obtained using magneto-optical techniques, to understand how to interpret them correctly [3].

Corresponding author: ZhenshengTao@fudan.edu.cn 


\section{Experimental results}

A schematic of the experimental setup is shown in Fig. 1. The sample used in our experiments was a $400 \mathrm{~nm} \mathrm{Ni(111)} \mathrm{single-crystalline} \mathrm{film.} \mathrm{In} \mathrm{both} \mathrm{the} \mathrm{Tr-TMOKE} \mathrm{and} \mathrm{Tr-}$ ARPES experiments, the sample was excited by $\sim 45 \mathrm{fs}$ pulses at a wavelength of $800 \mathrm{~nm}$. In the Tr-TMOKE measurements, the subsequent change of the sample magnetization was probed by magneto-optical Kerr effect with EUV pulses produced by high harmonic generation (HHG). In the Tr-ARPES measurements, the magnetization dynamics was probed by monitoring the change of exchange splitting at different time delays. The pump penetration depth in $\mathrm{Ni}$ is $\delta_{L} \sim 13 \mathrm{~nm}$, which is comparable to the probing depth of the EUV light used in the Tr-TMOKE experiments. In contrast, the probing depth of photoelectrons is close to a monolayer for the photon energy $(\sim 16 \mathrm{eV})$ used in the Tr-ARPES experiments.

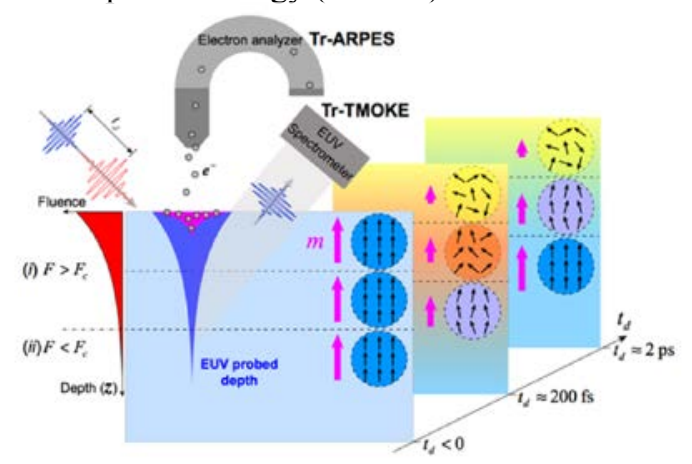

Figure 1. Schematic of EUV Tr-ARPES and TMOKE measurements on $\mathrm{Ni}(111)$.

The difference in the probing depths of the two methods is highlighted.

Due to $<1 \mathrm{~nm}$ probing depth, Tr-ARPES probes the elementary magnetization dynamics in a monolayer of the material, which can be described as:

$$
m\left(t_{d}, z\right)= \begin{cases}1 & \left(t_{d}<0\right) . \\ 1+a_{1}(z) e^{-\frac{t_{d}}{\tau_{\text {demag }}}}-a_{2}(z) e^{-\frac{t_{d}}{\tau_{\text {recourer }}}}-a_{3}(z) e^{-\frac{t_{d}}{\tau_{\text {reover } 2}}} & \left(t_{d} \geq 0\right)\end{cases}
$$

We directly extract from the Tr-ARPES measurements the three time constants that correspond to the following physical processes [2]: the collapse of the exchange splitting $\tau_{\text {demag }}=176 \pm 27 \mathrm{fs}$; a fast recovery time $\tau_{\text {recover } 1}=537 \pm 173 \mathrm{fs}$; and a slow recovery time $\tau_{\text {recover } 2}$ $=76 \pm 15$ ps. In Eq. (1), $a_{1}, a_{2}$ and $a_{3}$ are the amplitudes of these processes, with $a_{1}=a_{2}+a_{3}$. Their values depend on the strength of the laser fluence, and, hence, are depth dependent.

The in-situ laser fluence $F$ decays exponentially with the depth $z$, i.e., $F(z)=F_{0} e^{-z / \delta_{L}}$, where $F_{0}$ is the fluence at the surface. Here, we further assume that the change of magnetization is a linear function of the in-situ fluence:

and

$$
a_{1}(z)=\min \left[b_{1} F(z), 1\right]
$$

$$
a_{3}(z)= \begin{cases}0 & {\left[F(z)<F_{c}\right]} \\ \min \left\{b_{3}\left[F(z)-F_{c}\right], 1\right\} & {\left[F(z) \geq F_{c}\right]}\end{cases}
$$

where $b_{1}$ and $b_{3}$ are the proportionality constants. The Tr-TMOKE signals can hence be modelled as the bulk-averaged magnetization:

$$
\langle M\rangle\left(t_{d}\right)=\frac{\int_{0}^{\infty} m\left(t_{d}, z\right) W(z) \mathrm{d} z}{\int_{0}^{\infty} W(z) \mathrm{d} z}
$$

Here $W(z)$ is the depth sensitivity function of TMOKE, which can be calculated for Ni [4].

Using the model described above, we fit the Tr-TMOKE results under the different fluences shown in Fig. 2 to Eq. (1-4), taking only $b_{1}, b_{3}$ and $F_{\mathrm{c}}$ as the fitting parameters. 
The agreement between the model (solid red lines) and experimental data (symbols) is excellent over the full range of pump fluences, even though the limited number of fitting parameters places a strong constraint on our fitting. In the inset of Fig. 2, we plot a complete temporal and spatial profile of the laser-induced ultrafast demagnetization in $\mathrm{Ni}$.

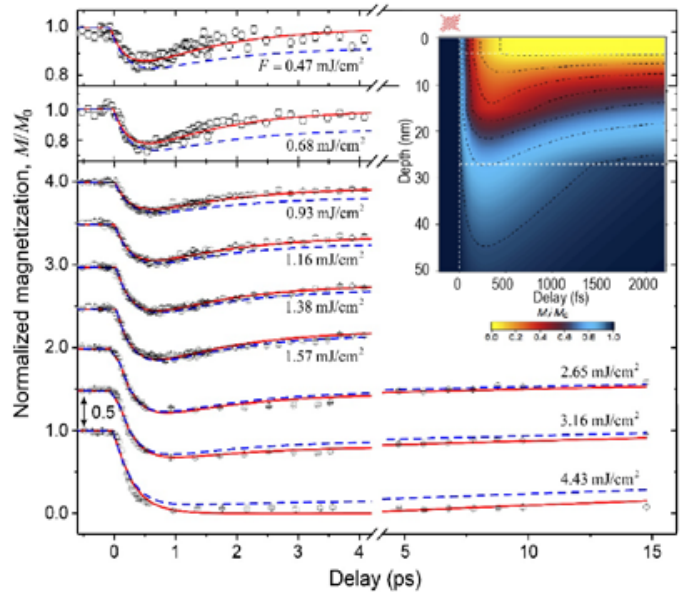

Figure 2. Magnetization dynamics in Ni measured using Tr-TMOKE over a full range of laser fluences. Red solid lines: fits to microscopic model using Eq. (1-4) which considers the critical behavior. Dashed blue lines: fits to the microscopic model without the critical behavior. Inset: The laser-induced magnetization variation in $\mathrm{Ni}$ as a function of time and depth under a pump fluence $\sim 5 \mathrm{~mJ} / \mathrm{cm}^{2}$.

\section{Discussion and conclusion}

From the results, we find the apparent presence of a fluence-dependent remagnetization time, which were regarded as important evidence for various microscopic mechanisms. In contrast, we explain this observation by considering the bulk-averaging effect in Tr-TMOKE: the surface layers of the material undergo a phase transition and exhibit slow recovery dynamics, while layers deeper within the material do not undergo a magnetic phase transition and as a result, exhibit only fast recovery dynamics.

We also clearly observed the competition between the fast and slow recovery dynamics, which can be attributed to distinct physical mechanisms. The fast recovery timescale $\left(\tau_{\text {recoverl }}\right)$ can be explained by damping of magnons under the strong exchange field in ferromagnetic $\mathrm{Ni}$, while the slow recovery $\left(\tau_{\text {recover } 2}\right)$ must arise from coupling to lattice and thermal transport after the sample enters the paramagnetic phase. Our results, hence, suggest the competition and coexistence of paramagnetic and partially suppressed ferromagnetic phases during the ultrafast demagnetization process.

We gratefully acknowledge support from the US DOE X-Ray Scattering Program for the magnetic spectroscopy measurements, and the JILA PFC and Moore Foundation EPiQS Program for the ARPES measurements.

\section{References}

1. E. Beaurepaire, J.-C. Merle, A. Daunois, and J.-Y. Bigot, Phys. Rev. Lett. 76, 4250 (1996).

2. P. Tengdin et al., Sci. Adv. 4, eaap9744 (2018).

3. W. You et al., Phys. Rev. Lett. in press (2018)

4. G. Traeger, L. Wenzel, and A. Hubert, Phys. Stat. Sol. 131, 201 (1992). 